\title{
CRITICAL POINT THEOREMS
}

\section{H. BOUKHRISSE and M. MOUSSAOUI}

Received 12 December 2000

Let $H$ be a Hilbert space such that $H=V \oplus W$, where $V$ and $W$ are two closed subspaces of $H$. We generalize an abstract theorem due to Lazer et al. (1975) and a theorem given by Moussaoui (1990-1991) to the case where $V$ and $W$ are not necessarily finite dimensional. We give two mini-max theorems where the functional $\Phi: H \rightarrow \mathbb{R}$ is of class $\mathscr{b}^{2}$ and $\mathscr{b}^{1}$, respectively.

2000 Mathematics Subject Classification: 58E05.

1. Introduction. Our purpose in this note is to generalize a mini-max theorem due to Lazer et al. [3]. Their theorem is as follows.

THEOREM 1.1. Let $X$ and $Y$ be two closed subspaces of a real Hilbert space $H$ such that $X$ is finite dimensional and $H=X \oplus Y$ ( $X$ and $Y$ not necessarily orthogonal). Let $\Phi: H \rightarrow \mathbb{R}$ be a $C^{2}$ functional and let $\nabla \Phi$ and $D^{2} \Phi$ denote the gradient and Hessian of $\Phi$, respectively. Suppose that there exist two positive constants $m_{1}$ and $m_{2}$ such that

$$
\left(D^{2} \Phi(u) h, h\right) \leq-m_{1}\|h\|^{2}, \quad\left(D^{2} \Phi(u) k, k\right) \geq m_{2}\|k\|^{2}
$$

for all $u \in H, h \in X$, and $k \in Y$. Then $\Phi$ has a unique critical point, that is, there exists a unique $v_{0} \in H$ such that $\nabla \Phi\left(v_{0}\right)=0$. Moreover, this critical point is characterized by the

$$
\Phi\left(v_{0}\right)=\max _{x \in X} \min _{y \in Y} \Phi(x+y) .
$$

Bates and Ekeland in [1] generalized Theorem 1.1 to the case where $X$ and $Y$ are not necessarily finite dimensional. Via a reduction method, Manasevich considered the same case in [4], but he supposed weaker conditions on Hessian of $\Phi$. On the other hand, Tersian [7] studied the case where $X$ and $Y$ are not necessarily finite dimensional, $\nabla \Phi: H \rightarrow H$ is everywhere defined and hemicontinuous on $H$, which means that

$$
\lim _{t \rightarrow 0} \nabla \Phi(u+t v)=\nabla \Phi(u) \quad \forall u, v \in H .
$$

Instead of the conditions on the Hessian of $\Phi$, they supposed

(1) $\left(\nabla \Phi\left(h_{1}+y\right)-\nabla \Phi\left(h_{2}+y\right), h_{1}-h_{2}\right) \leq-m_{1}\left\|h_{1}-h_{2}\right\|^{2} h_{1}, h_{2} \in X, y \in Y$,

(2) $\left(\nabla \Phi\left(x+k_{1}\right)-\nabla \Phi\left(x+k_{2}\right), k_{1}-k_{2}\right) \geq m_{2}\left\|k_{1}-k_{2}\right\|^{2} k_{1}, k_{2} \in Y, x \in X$, where $H=X \oplus Y, m_{1}$ and $m_{2}$ are strictly positive.

Their result rests heavily upon two theorems on $\alpha$-convex functionals and an existence theorem for a class of monotone operators due to Browder. By a completely 
different method, the second author gave another version of Theorem 1.1 (see [5]) with convexity conditions that are weaker than those assumed above.

THEOREM 1.2. Let $H$ be a Hilbert space such that $H=V \oplus W$ where $V$ is a finitedimensional subspace of $H$ and $W$ its orthogonal. Let $\Phi: H \rightarrow \mathbb{R}$ be a functional such that

(i) $\Phi$ is of class $\mathscr{C}^{1}$.

(ii) $\Phi$ is coercive on $W$.

(iii) For fixed $w \in W, v \mapsto \Phi(v+w)$ is concave on $V$.

(iv) For fixed $w \in W, \Phi(v+w) \rightarrow-\infty$ when $\|v\| \rightarrow+\infty, v \in V$; and the convergence is uniform on bounded subsets of $W$.

(v) For all $v \in V$, $\Phi$ is weakly lower semicontinuous on $W+v$.

Then $\Phi$ admits a critical point in $H$.

We consider the case where $X$ and $Y$ are not necessarily finite dimensional. Our proofs contain many steps used in [5] and our convexity conditions are weaker than those given by other authors. First, we prove a mini-max theorem where $\Phi: H \rightarrow \mathbb{R}$ is of class $\mathfrak{C}^{2}$. Next, we prove the existence theorem for a particular class of $\mathscr{C}^{1}$ functional $\Phi: H \rightarrow \mathbb{R}$.

2. First abstract result. The next two propositions are used in this work. For a proof of Proposition 2.1, see [2], and for a proof of Proposition 2.2, see [6].

Proposition 2.1. Let $X$ be a reflexive Banach space and let $\Phi: X \rightarrow \mathbb{R}$ be a functional such that

(i) $\Phi$ is weakly lower semicontinuous on $X$,

(ii) $\Phi$ is coercive, that is, $\Phi(u) \rightarrow+\infty$ when $\|u\| \rightarrow+\infty$, then $\Phi$ is lower bounded and there exists $u_{0} \in X$ such that

$$
\Phi\left(u_{0}\right)=\inf _{X} \phi .
$$

Proposition 2.2. Let $H$ be a real Hilbert space and let $L$ be a bounded linear operator on $H$. Suppose that

$$
(L x, x) \geq a\|x\|^{2},
$$

for all $x \in H$ and $a$ is a strictly positive real number. Then $L$ is an isomorphism onto $H$ and $\left\|L^{-1}\right\| \leq a^{-1}$.

THEOREM 2.3. Let $H$ be a Hilbert space such that $H=V \oplus W$ where $V$ and $W$ are two closed and orthogonal subspaces of $H$. Let $\Phi: H \rightarrow \mathbb{R}$ be a functional such that

(i) $\Phi$ is of class $\mathscr{C}^{2}$.

(ii) There exists a continuous nonincreasing function $\gamma:[0,+\infty) \rightarrow] 0, \infty)$ such that

$$
\left\langle D^{2} \Phi(v+w) g, g\right\rangle \leq-\gamma(\|v\|)\|g\|^{2}
$$

for all $v \in V, w \in W$, and $g \in V$.

(iii) $\Phi$ is coercive on $W$.

(iv) For all $w \in W, \Phi(v+w) \rightarrow-\infty$ when $\|v\| \rightarrow+\infty, v \in V$.

(v) $\Phi$ is weakly lower semicontinuous on $W+v$. 
Then $\Phi$ admits at least a critical point $u \in H$. Moreover, this critical point of $\Phi$ is characterized by the equality

$$
\Phi(u)=\min _{w \in W} \max _{v \in V} \Phi(v+w) .
$$

In the proof of Theorem 2.3, we will use the following three lemmas.

LEMMA 2.4. For all $w \in W$, there exists a unique $v \in V$ such that

$$
\Phi(v+w)=\max _{g \in V} \Phi(g+w) .
$$

Proof. From Theorem 2.3(ii), for $w$ fixed in $W, v \mapsto \Phi(v+w)$ is continuous and strictly concave on $V$. Then, it is weakly upper semicontinuous on $V$. Moreover, from Theorem 2.3(iv), it is anticoercive on $V$. So that it admits a maximum on $V$. We affirm that this maximum is unique, otherwise we suppose that there exists two maximums $v_{1}$ and $v_{2}$. Let $v_{\lambda}=\lambda v_{1}+(1-\lambda) v_{2}$ for $0<\lambda<1$, then

$$
\Phi\left(v_{\lambda}+w\right)>\lambda \Phi\left(v_{1}+w\right)+(1-\lambda) \Phi\left(v_{2}+w\right)=\Phi\left(v_{1}+w\right)=\Phi\left(v_{2}+w\right) .
$$

For the rest of the note, we will adopt the notations

$$
\begin{gathered}
\bar{V}(w)=\left\{v \in V: \Phi(v+w)=\max _{g \in V} \Phi(g+w)\right\}, \\
S=\{u=v+w, w \in W, v \in \bar{V}(w)\} .
\end{gathered}
$$

LEMMA 2.5. There exists $u \in S$ such that

$$
\Phi(u)=\inf _{S} \Phi .
$$

Proof. There exists a sequence $\left(u_{n}\right)$ of $S$ such that $\Phi\left(u_{n}\right) \rightarrow \inf _{S} \Phi=a$. For all $n$, $u_{n}=v_{n}+w_{n}$ with $w_{n} \in W$, and $v_{n} \in \bar{V}\left(w_{n}\right)$.

Claim

$$
\left\|w_{n}\right\| \leq c
$$

Otherwise,

$$
\Phi\left(u_{n}\right)=\Phi\left(v_{n}+w_{n}\right) \geq \Phi\left(w_{n}\right) .
$$

From Theorem 2.3(iii), $\Phi\left(w_{n}\right) \rightarrow+\infty$, hence $\Phi\left(u_{n}\right) \rightarrow+\infty$. This gives a contradiction. Moreover, from (2.9), there exists a subsequence also denoted $w_{n}$ such that $w_{n} \rightarrow w$. Take $v$ in $V$, by Theorem 2.3(v), we have

$$
\Phi(v+w) \leq \liminf _{n} \Phi\left(v+w_{n}\right) \leq \liminf _{n} \Phi\left(v_{n}+w_{n}\right)=a .
$$

This is true for all $v \in V$, in particular, for $v \in \bar{V}(w)$. Then $u=v+w$ satisfies (2.8).

LEMmA 2.6. The application $\bar{V}: W \rightarrow V$ such that

$$
\Phi(w+\bar{V}(w))=\max _{g \in V} \Phi(g+w)
$$

is of class $C^{1}$. 
Proof of Theorem 2.3. For each $w \in W$, let $\Phi_{w}: V \rightarrow \mathbb{R}$ be defined by $\Phi_{w}(v)=$ $\Phi(v+w)$. Then $\Phi_{w} \in C^{2}(V, \mathbb{R})$ and for $v^{\prime} \in V$, we have

$$
\begin{aligned}
\left(\nabla \phi_{w}(v), v^{\prime}\right) & =\left(\nabla \Phi(v+w), v^{\prime}\right), \\
\left(D^{2} \Phi_{w}(v) v^{\prime}, v^{\prime}\right) & =\left(D^{2} \Phi(v+w) v^{\prime}, v^{\prime}\right) .
\end{aligned}
$$

By Lemma 2.4, we conclude that for all $w \in W$, there exists a unique $v_{w}$ in $V$ such that $\nabla \Phi_{w}\left(v_{w}\right)=0$. To prove that $\bar{V} \in C^{1}(W, V)$, we will use the implicit function theorem. To see this, let $P$ denote the orthogonal projection of $H$ onto $V$. Then

$$
v=\bar{V}(w) \quad \text { iff } P \nabla \Phi(w+v)=0 .
$$

Next, we define $E: W \times V \rightarrow V$ by

$$
E(w, v)=P \nabla \Phi(w+v) .
$$

Then $E$ is of class $C^{1}$ and given any pair $w_{0} \in W, v_{0} \in V$ such that $E\left(w_{0}, v_{0}\right)=0$, it follows that $v_{0}=\bar{V}\left(w_{0}\right)$.

If $E_{v}$ denotes the partial derivative of $E$ with respect to $v$, and if $v^{\prime} \in V$, we have

$$
E_{v}\left(w_{0}, v_{0}\right) v^{\prime}=P D^{2} \Phi\left(w_{0}+v_{0}\right) v^{\prime}
$$

The mapping $E_{v}\left(w_{0}, v_{0}\right): V \rightarrow V$ is linear and bounded we have from Theorem 2.3(ii)

$$
\left(E_{v}\left(w_{0}, v_{0}\right) v^{\prime}, v^{\prime}\right)=\left(D^{2} \Phi\left(w_{0}+v_{0}\right) v^{\prime}, v^{\prime}\right) \leq-\gamma\left(\left\|v_{0}\right\|\right)\left\|v^{\prime}\right\|^{2},
$$

for all $v^{\prime} \in V$. By Proposition 2.2, $E_{v}\left(w_{0}, v_{0}\right)$ is an isomorphism onto $V$. Then from the implicit function theorem [2], there exists a $C^{1}$ mapping $f$ from a neighborhood $U$ of $w_{0}$ in $W$ into $V$ such that $E(w, f(w))=0$ for all $w \in U$. Moreover, from (2.14) and (2.15), $f(w)=\bar{V}(w)$ for all $w \in W$. Hence, since $w_{0}$ was arbitrarily chosen, it follows that $f$ can be defined over all of $W$. Then we conclude that $\bar{V} \in C^{1}(W, V)$.

REMARK 2.7. The proof of Lemma 2.6 relies on the implicit function theorem. This theorem was used by Thews in [8] to prove the existence of a critical point for a particular class of functionals. It was also used by Manasevich in [4].

Proof. Let $w \in W$ and $u \in S_{w}$. We will prove that if $u$ satisfies (2.8), then $u$ is a critical point of $\Phi$. By Lemma 2.4 , it is easy to see that $(\nabla \Phi(u), g)=0$ for all $g \in V$, so it suffices to prove that

$$
(\nabla \Phi(u), h)=0 \quad \forall h \in W
$$

Recall that $u \in S$ can be written $u=w+v$ where $w \in W$ and $v \in \bar{V}(w)$. Take $h \in W$ and let $w_{t}=w+t h$ for $|t| \leq 1$. For each $t$ such that $0<|t| \leq 1$, there exists a unique $v_{t} \in V\left(w_{t}\right)$. By Lemma 2.6, we conclude that $v_{t_{n}}$ converge to a certain $v_{0}$ and that $v_{0} \in \bar{V}(w)$. Then, by Lemma 2.4, $v_{0}=v$. For $t>0$, we have

$$
\frac{\Phi\left(w_{t}+v_{t}\right)-\Phi\left(v_{t}+w\right)}{t} \geq \frac{\Phi\left(w_{t}+v_{t}\right)-\Phi\left(v_{0}+w\right)}{t} \geq 0
$$


Then,

$$
\left(\nabla \Phi\left(v_{t}+w+\lambda_{t} t h\right), h\right) \geq 0 \text { for } 0<\lambda_{t}<1 .
$$

At the limit, we obtain

$$
(\nabla \Phi(u), h)=0 \quad \forall h \in W
$$

Hence, $u$ is a critical point of $\Phi$.

3. Second abstract result. Let $H$ be a Hilbert space such that $H=V \oplus W$ where $V$ and $W$ are two closed and orthogonal subspaces of $H$. Let $\Phi: H \rightarrow \mathbb{R}$ be such that

$$
\begin{gathered}
\Phi=q+\psi, \\
q(v+w)=q(v)+q(w) \quad \forall(v, w) \in V \times W \\
\psi \text { is weakly continuous on } H .
\end{gathered}
$$

THEOREM 3.1. Let $H$ be a Hilbert space such that $H=V \oplus W$ where $V$ and $W$ are two closed and orthogonal subspaces of $H$. Let $\Phi: H \rightarrow \mathbb{R}$ be a functional verifying (3.1) such that

(i) $q$ and $\psi$ are of class $\mathfrak{b}^{1}$.

(ii) $\nabla \Phi$ is weakly continuous on $H$.

(iii) $\Phi$ is coercive on $W$.

(iv) For a fixed $w \in W, v \mapsto \Phi(v+w)$ is concave on $V$.

(v) For a fixed $w \in W, \Phi(v+w) \rightarrow-\infty$ when $\|v\| \rightarrow+\infty, v \in V$; and the convergence is uniform on the bounded sets of $W$.

(vi) For a fixed $v \in V$, $\Phi$ is weakly lower semicontinuous on $W+v$.

Then $\Phi$ admits a critical point $u \in H$. Moreover, this critical point is characterized by the equality

$$
\Phi(u)=\min _{w \in W} \max _{v \in V} \Phi(v+w) .
$$

For the proof of Theorem 3.1, we use some results of Lemmas 2.4 and 2.5 and we need also the following lemmas.

LEMMA 3.2. For each $w \in W, \bar{V}(w)$ is convex.

Proof. Take $v_{1}, v_{2} \in \bar{V}(w)$ and $v_{\lambda}=\lambda v_{1}+(1-\lambda) v_{2}$ with $\lambda \in[0,1]$. So that from Theorem 3.1(iv), we have $\Phi\left(v_{\lambda}+w\right) \geq \lambda \Phi\left(v_{1}+w\right)+(1-\lambda) \Phi\left(v_{2}+w\right)=\Phi\left(v_{1}+w\right)=$ $\Phi\left(v_{2}+w\right)$. Then

$$
\Phi\left(v_{\lambda}+w\right)=\Phi\left(v_{1}+w\right)=\Phi\left(v_{2}+w\right)
$$

So $v_{\lambda} \in \bar{V}(w)$.

LEMmA 3.3. Let $L(w)=\{\nabla \Phi(v+w): v \in \bar{V}(w)\}$. For each $w \in W$,

(i) $L(w)$ is convex.

(ii) $L(w)$ is closed. 
Proof. (i) Let $h \in W$ and $v_{1}, v_{2} \in \bar{V}(w)$. From Theorem 3.1(iv) and Lemma 3.2, we have for all $t>0$,

$$
\begin{aligned}
\Phi\left(v_{\lambda}+w+t h\right)-\Phi\left(v_{\lambda}+w\right) \geq & \lambda\left(\Phi\left(v_{1}+t h+w\right)-\Phi\left(v_{1}+w\right)\right) \\
& +(1-\lambda)\left(\Phi\left(v_{2}+t h+w\right)-\Phi\left(v_{2}+w\right)\right) .
\end{aligned}
$$

Divide by $t$ and let $t$ tend to 0 , then

$$
\left(\nabla \Phi\left(v_{\lambda}+w\right), h\right) \geq \lambda\left(\nabla \Phi\left(v_{1}+w\right), h\right)+(1-\lambda)\left(\nabla \Phi\left(v_{2}+w\right), h\right) .
$$

Since this is true for all $h \in W$, we conclude that

$$
\nabla \Phi\left(v_{\lambda}+w\right)=\lambda \nabla \Phi\left(v_{1}+w\right)+(1-\lambda) \nabla \Phi\left(v_{2}+w\right)
$$

(ii) For $w \in W$, let $S_{w}=\{v+w: v \in \bar{V}(w)\}$.

First, we show that $S_{w}$ is closed. Let $v_{n}+w \in S_{w}$ such that $v_{n}+w \rightarrow v_{0}+w . \Phi\left(v_{n}+\right.$ $w) \rightarrow \Phi\left(v_{0}+w\right)$ and $\Phi\left(v_{n}+w\right)=\max _{g \in V} \Phi(g+w)$. Then $v_{0}+w \in S_{w}$.

Next, we affirm that $S_{w}$ is bounded. If not, there exists $v_{n}$ of $\bar{V}(w)$ such that $\left\|v_{n}\right\| \rightarrow+\infty$, and we conclude from Theorem 3.1(v) that $\Phi\left(v_{n}+w\right) \rightarrow-\infty$. This gives a contradiction.

Consequently, $S_{w}$ is closed and bounded. Since $S_{w}$ is convex, we conclude that $S_{w}$ is weakly compact. From Theorem 3.1(ii), it follows that $L(w)$ is weakly compact. Then $L(w)$ is weakly closed. Thus $L(w)$ is closed.

Proof of TheOrem 3.1. Let $w \in W$ and $u \in S_{w}$. If $u$ satisfies (2.8), we will show that $L(w)$ contains 0 and there exists $v \in \bar{V}(w)$ such that

$$
\nabla \Phi(v+w)=0
$$

By contradiction, suppose that $L(w)$ does not contain 0 . Since it is convex and closed in the Hilbert space, there exists $h_{1} \in L(w)$ such that

$$
0 \neq\left\|h_{1}\right\|=\inf \{\|h\|: h \in L(w)\} .
$$

Let $h \in L(w), h_{1}+\lambda\left(h-h_{1}\right) \in L(w)$ for $\lambda \in[0,1]$, thus

$$
\left(h_{1}+\lambda\left(h-h_{1}\right), h_{1}+\lambda\left(h-h_{1}\right)\right) \geq\left\|h_{1}\right\|^{2} .
$$

Hence

$$
\left\|h_{1}\right\|^{2}+2 \lambda\left(h-h_{1}, h_{1}\right)+\lambda^{2}\left\|h-h_{1}\right\|^{2} \geq\left\|h_{1}\right\|^{2},
$$

so

$$
2\left(h-h_{1}, h_{1}\right)+\lambda\left\|h-h_{1}\right\|^{2} \geq 0 .
$$

When $\lambda$ tends to 0 . We obtain $\left(h-h_{1}, h_{1}\right) \geq 0$. So that $\left(h, h_{1}\right) \geq\left\|h_{1}\right\|^{2}>0$. Equivalently,

$$
\left(\nabla \Phi(v+w), h_{1}\right)>0 \quad \forall v \in \bar{V}(w) .
$$


Denote $w_{t}=w+t h_{1}$ for $|t| \leq 1$. We note that $w_{t} \in W$. By Lemma 2.4, for each $0<$ $|t| \leq 1$, there exists $v_{t} \in V\left(w_{t}\right)$. Since $\left\|w_{t}\right\| \leq\|w\|+\left\|h_{1}\right\|$, Theorem 3.1(v) implies that there exists a constant $A>0$ such that

$$
\Phi\left(v+w_{t}\right)<\inf _{W} \Phi \leq \Phi\left(w_{t}\right),
$$

for $v \in V,\|v\| \geq A$, and $|t| \leq 1$. (Since $\Phi$ is coercive and weakly lower semicontinuous in the reflexive space $W$, it reaches its minimum.) It follows that

$$
\left\|v_{t}\right\| \leq A
$$

Otherwise, we would have

$$
\Phi\left(v_{t}+w_{t}\right)<\Phi\left(w_{t}\right)
$$

which contradicts the fact that $v_{t} \in \bar{V}\left(w_{t}\right)$. We conclude then as $V$ is reflexive that there exists a subsequence $t_{n} \rightarrow 0$ and $t_{n}<0$ such that $v_{t_{n}} \rightarrow v_{0} \in V$.

Claim

$$
v_{0} \in \bar{V}(w)
$$

We have $v_{t_{n}} \rightarrow v_{0}$ and $w_{t_{n}} \rightarrow w$, so

$$
v_{t_{n}}+w_{t_{n}}-v_{0}+w
$$

Since $\psi$ is weakly upper semicontinuous on $H$, we have

$$
\psi\left(v_{0}+w\right) \geq \limsup _{n \rightarrow \infty} \psi\left(v_{t_{n}}+w_{t_{n}}\right) .
$$

By Lemma 2.4, $\Phi$ is weakly upper semicontinuous on $V$ and we know that $\psi$ is weakly lower semicontinuous on $V$, so $q=\Phi-\psi$ is weakly upper semicontinuous on $V$. Then

$$
q\left(v_{0}\right) \geq \limsup _{n \rightarrow \infty} q\left(v_{t_{n}}\right) .
$$

Moreover, the continuity of $q$ implies that

$$
q(w)=\lim _{n \rightarrow \infty} q\left(w_{t_{n}}\right)=\limsup _{n \rightarrow \infty} q\left(w_{t_{n}}\right) .
$$

Then

$$
\begin{aligned}
q\left(v_{0}+w\right) & =q\left(v_{0}\right)+q(w) \\
& \geq \limsup _{n \rightarrow \infty} q\left(v_{t_{n}}\right)+\limsup _{n \rightarrow \infty} q\left(w_{t_{n}}\right) \\
& \geq \limsup _{n \rightarrow \infty}\left(q\left(v_{t_{n}}\right)+q\left(w_{t_{n}}\right)\right) \\
& \geq \limsup _{n \rightarrow \infty} q\left(v_{t_{n}}+w_{t_{n}}\right) .
\end{aligned}
$$

On the other hand, $v_{t_{n}} \in V\left(w_{t_{n}}\right)$ implies that

$$
\Phi\left(v_{t_{n}}+w_{t_{n}}\right) \geq \Phi\left(v+w_{t_{n}}\right) \quad \forall v \in V .
$$


We then obtain

$$
\begin{aligned}
q\left(v_{0}+w\right)+\psi\left(v_{0}+w\right) & \geq \limsup _{n \rightarrow \infty} q\left(v_{t_{n}}+w_{t_{n}}\right)+\limsup _{n \rightarrow \infty} \psi\left(v_{t_{n}}+w_{t_{n}}\right) \\
& \geq \limsup _{n \rightarrow \infty}\left(q\left(v_{t_{n}}+w_{t_{n}}\right)+\psi\left(v_{t_{n}}+w_{t_{n}}\right)\right) \\
& \geq \limsup _{n \rightarrow \infty}\left(q\left(v+w_{t_{n}}\right)+\psi\left(v+w_{t_{n}}\right)\right) \quad \forall v \in V \\
& \geq q(v+w)+\psi(v+w) \quad \forall v \in V .
\end{aligned}
$$

Thus

$$
\Phi\left(v_{0}+w\right) \geq \Phi(v+w) \quad \forall v \in V .
$$

Equivalently, $v_{0} \in \bar{V}(w)$.

Therefore, we have

$$
-\frac{\Phi\left(w_{t_{n}}+v_{t_{n}}\right)-\Phi\left(v_{t_{n}}+w\right)}{t_{n}} \geq-\frac{\Phi\left(w_{t_{n}}+v_{t_{n}}\right)-\Phi\left(v_{0}+w\right)}{t_{n}} \geq 0,
$$

and so

$$
\left(\nabla \Phi\left(v_{t_{n}}+w+\varepsilon_{n} t_{n} h_{1}\right), h_{1}\right) \leq 0 \text { for } 0<\varepsilon_{n}<1 .
$$

When $t_{n}$ tend to 0 , by (ii), we deduce finally that

$$
\left(\nabla \Phi\left(v_{0}+w\right), h_{1}\right) \leq 0 .
$$

Which contradicts (3.8). Then there exists $v_{1} \in \bar{V}(w)$ such that $\nabla \Phi\left(v_{1}+w\right)=0$ and

$$
\Phi\left(v_{1}+w\right)=\min _{w \in W} \max _{v \in V} \Phi(v+w) .
$$

REMARK 3.4. In the proof of Theorem 3.1, (3.1) allows us to show that $v_{0} \in \bar{V}(w)$. Or, we remark that we do not need to introduce $\psi$ and $q$ if $\Phi(v+w)=\Phi(v)+\Phi(w)$. Indeed, $w_{t_{n}} \rightarrow w$ and $v_{t_{n}} \rightarrow v_{0}$ imply that

$$
\begin{aligned}
\limsup \Phi\left(v_{t_{n}}+w_{t_{n}}\right) & =\limsup \left(\Phi\left(v_{t_{n}}\right)+\Phi\left(w_{t_{n}}\right)\right) \\
& \leq \limsup \Phi\left(v_{t_{n}}\right)+\lim \sup \Phi\left(w_{t_{n}}\right) .
\end{aligned}
$$

By Lemma 2.4, $\Phi$ is weakly upper semicontinuous on $V$, thus

$$
\limsup \Phi\left(v_{t_{n}}+w_{t_{n}}\right) \leq \Phi\left(v_{0}\right)+\Phi(w)=\Phi\left(v_{0}+w\right) .
$$

On the other hand, $v_{t_{n}} \in \bar{V}\left(w_{t_{n}}\right)$ implies that

$$
\Phi\left(v_{t_{n}}+w_{t_{n}}\right) \geq \Phi\left(v+w_{t_{n}}\right) \quad \forall v \in V .
$$

So

$$
\limsup \Phi\left(v_{t_{n}}+w_{t_{n}}\right) \geq \Phi(v+w) \quad \forall v \in V
$$

Then

$$
\Phi\left(v_{0}+w\right) \geq \Phi(v+w) \quad \forall v \in V,
$$

that is, $v_{0} \in \bar{V}(w)$. 
REMARK 3.5. We can also prove Theorem 3.1 for any functional $\Phi: H \rightarrow \mathbb{R}$ without introducing $\psi$ and $q$ if $\Phi$ is weakly upper semicontinuous on $H$.

ANOTHER VERSION OF THEOREM 3.1. Let $A$ be a convex set. The function $f: A \rightarrow \mathbb{R}$ is quasiconcave if for all $x_{1}, x_{2}$ in $A$, and for all $\lambda$ in $] 0,1[$, then

$$
f\left(\lambda x_{1}+(1-\lambda) x_{2}\right) \geq \min \left(f\left(x_{1}\right), f\left(x_{2}\right)\right) .
$$

The function $f$ is quasiconvex if $(-f)$ is quasiconcave, and it is strictly quasiconcave if the inequality above is strict.

It is clear that any strictly concave function is strictly quasiconcave.

Proposition 3.6. Let $E$ be a reflexive Banach space. If $\Phi: E \rightarrow \mathbb{R}$ is quasiconcave and upper semicontinuous, then $\Phi$ is weakly upper semicontinuous.

THeOrem 3.7. Let $E$ be a reflexive Banach space such that $E=V \oplus W$ where $V$ and $W$ are two closed subspaces of $E$ not necessarily orthogonal. Let $\Phi: H \rightarrow \mathbb{R}$ be a functional satisfying (3.1) such that

(i) $q$ and $\psi$ are of class $\mathscr{b}^{1}$.

(ii) $\nabla \Phi$ is weakly continuous.

(iii) $\Phi$ is coercive on $W$.

(iv) For all $w \in W, v \mapsto \Phi(v+w)$ is strictly quasiconcave on $V$.

(v) For all $w \in W, \Phi(v+w) \rightarrow-\infty$ when $\|v\| \rightarrow+\infty, v \in V$; and the convergence is uniform on bounded subsets of $W$.

(vi) For all $v \in V$, $\Phi$ is lower weakly semicontinuous on $W+v$.

Then $\Phi$ admits a critical point $u \in H$. Moreover, this critical point is characterized by the equality

$$
\Phi(u)=\min _{w \in W} \max _{v \in V} \Phi(v+w) .
$$

In the proof of this theorem, we need Lemmas 2.4 and 2.5. We note that by Proposition 3.6, the result of Lemma 2.4 is still true in this case.

Proof. We will prove that $u \in S$ obtained in Lemma 2.5 is a critical point of $\Phi$. We have $\left\langle\Phi^{\prime}(u), v\right\rangle$ for all $v \in V$, so it is sufficient to show that $\left\langle\Phi^{\prime}(u), h\right\rangle=0$ for all $h \in W$. Recall that $u \in S$ can be written as $u=v+w$ where $w \in W$ and $v \in \bar{V}(w)$. Let $h \in W$ and $w_{t}=w+t h$ for $|t| \leq 1$. For all $t$ such that $0<|t| \leq 1$, there exists a unique $v_{t} \in \bar{V}\left(w_{t}\right)$. In the same way as in the proof of Theorem 3.1, we can extract a subsequence $v_{t_{n}}$ such that $v_{t_{n}} \rightarrow v_{0}$ and $v_{0} \in \bar{V}(w)$. By Lemma 2.4, we deduce that $v_{0}=v$. Hence for $t>0$, we have

$$
\left\langle\Phi^{\prime}(u), h\right\rangle=0 \quad \forall h \in W .
$$

Then, $u$ is a critical point of $\Phi$.

\section{REFERENCES}

[1] P. W. Bates and I. Ekeland, A saddle-point theorem, Differential Equations (Proc. Eighth Fall Conf., Oklahoma State Univ., Stillwater, Okla., 1979), Academic Press, New York, 1980, pp. 123-126. 
[2] M. S. Berger, Nonlinearity and Functional Analysis. Lectures on Nonlinear Problems in Mathematical Analysis, Pure and Applied Mathematics, vol. 74, Academic Press, New York, 1977.

[3] A. C. Lazer, E. M. Landesman, and D. R. Meyers, On saddle point problems in the calculus of variations, the Ritz algorithm, and monotone convergence, J. Math. Anal. Appl. 52 (1975), no. 3, 594-614.

[4] R. F. Manasevich, A min max theorem, J. Math. Anal. Appl. 90 (1982), 64-71.

[5] A. M. Moussaoui, Questions d'existence dans les problèmes semi-lineaires elliptiques, Ph.D. thesis, Université libre de Bruxelles, 1990-1991.

[6] J. T. Schwartz, Nonlinear Functional Analysis, Gordon and Breach Science Publishers, New York, 1969.

[7] S. A. Tersian, A minimax theorem and applications to nonresonance problems for semilinear equations, Nonlinear Anal. 10 (1986), no. 7, 651-668.

[8] K. Thews, A reduction method for some nonlinear Dirichlet problems, Nonlinear Anal. 3 (1979), 795-813.

H. Boukhrisse: Department of MAThematics, FACUlty of Sciences, University MOHAMMED I, OUJDA, MOROCCO

E-mail address: boukhrisse@operamai 1.com

M. Moussaoui: Department of Mathematics, FAculty of Sciences, University MOHAMMED I, OUJDA, MOROCCO 


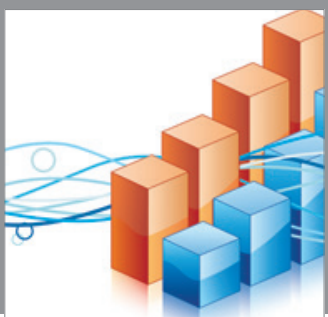

Advances in

Operations Research

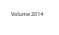

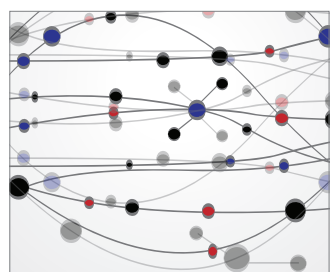

\section{The Scientific} World Journal
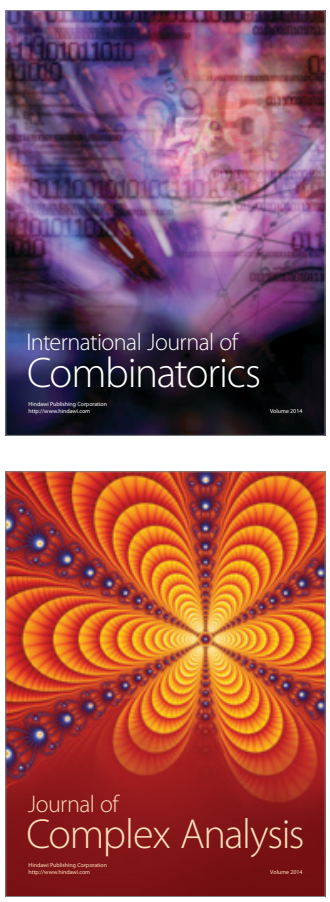

International Journal of

Mathematics and

Mathematical

Sciences
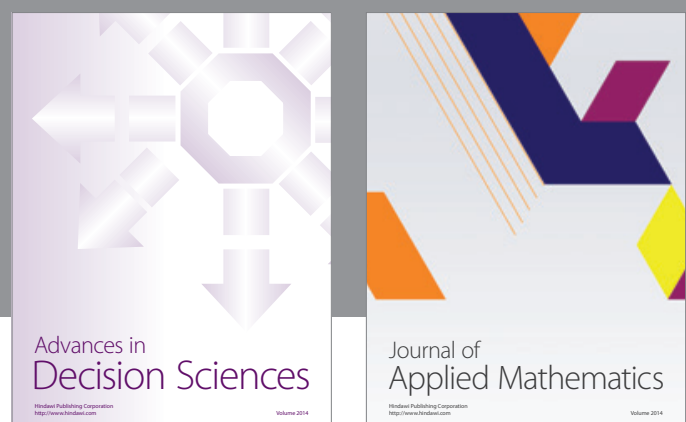

Journal of

Applied Mathematics
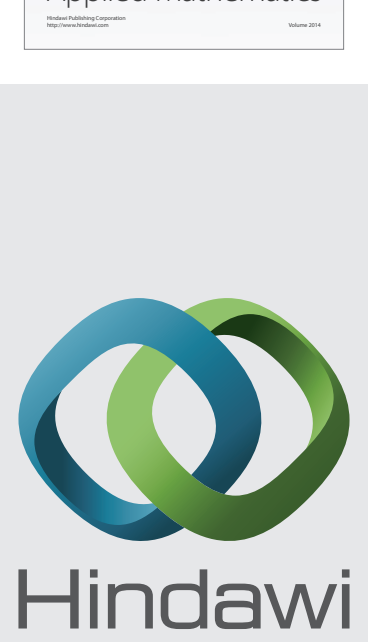

Submit your manuscripts at http://www.hindawi.com
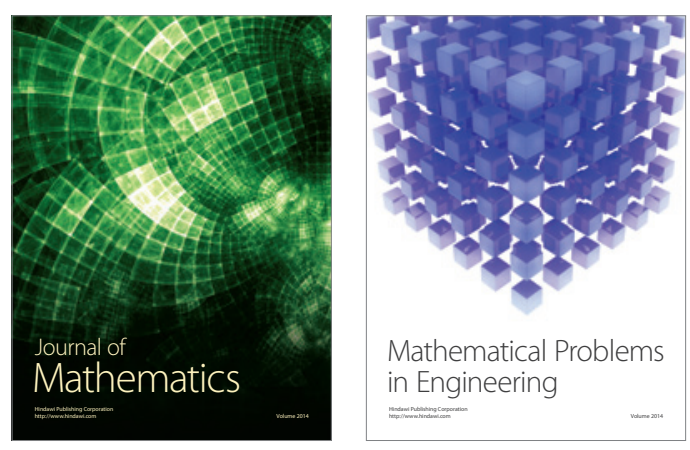

Mathematical Problems in Engineering
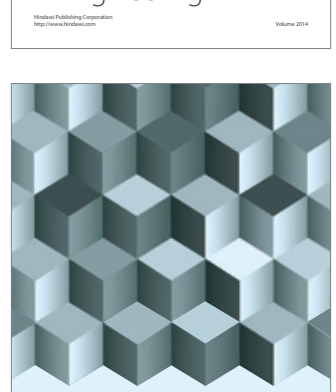

Journal of

Function Spaces
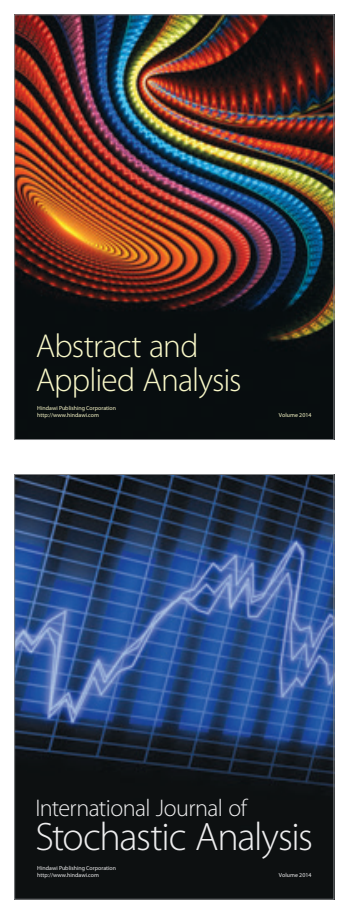

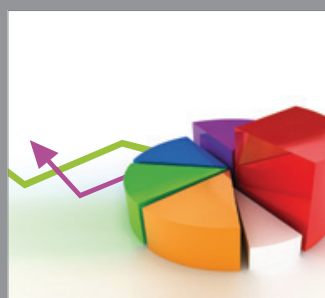

ournal of

Probability and Statistics

Promensencen
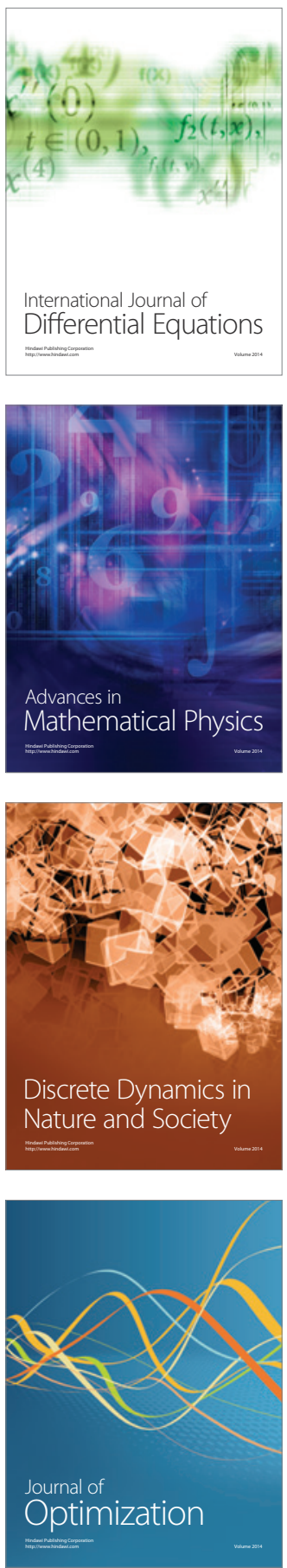